\title{
Structural characterization and functional properties of a novel lipomannan variant isolated from a Corynebacterium glutamicum pimB' ${ }^{\prime}$ mutant
}

\author{
Arun K. Mishra · Christina Klein · Sudagar S. Gurcha · Luke J. Alderwick • \\ Ponnusamy Babu · Paul G. Hitchen - Howard R. Morris · Anne Dell · \\ Gurdyal S. Besra $\cdot$ Lothar Eggeling
}

Received: 21 February 2008/Accepted: 4 April 2008/Published online: 18 April 2008

(C) The Author(s) 2008

\begin{abstract}
The genus Corynebacterium is part of the phylogenetic group nocardioform actinomycetes, which also includes the genus Mycobacterium. Members of this phylogenetic group have a characteristic cell envelope structure, which is dominated by complex lipids and amongst these, lipoglycans are of particular interest. The disruption of NCgl2106 in C. glutamicum resulted in a mutant devoid of monoacylated phosphatidyl-myo-inositol dimannoside $\left(\mathrm{Ac}_{1} \mathrm{PIM}_{2}\right)$ resulting in the accumulation of $\mathrm{Ac}_{1} \mathrm{PIM}_{1}$ and cessation of phosphatidyl-myo-inositol (PI) based lipomannan (Cg-LM, now also termed ' $\mathrm{Cg}-\mathrm{LM}-\mathrm{A}$ ') and lipoarabinomannan (Cg-LAM) biosynthesis. Interestingly, SDS-analysis of the lipoglycan fraction from the mutant revealed the synthesis of a single novel lipoglycan, now termed 'Cg-LM-B'. Further chemical analyses established
\end{abstract}

A. K. Mishra · S. S. Gurcha · L. J. Alderwick .

G. S. Besra ( $\square)$

School of Biosciences, University of Birmingham,

Edgbaston, Birmingham B15 2TT, UK

e-mail: g.besra@bham.ac.uk

C. Klein $\cdot$ L. Eggeling $(\bowtie)$

Institute for Biotechnology 1, Research Centre Juelich,

52425 Juelich, Germany

e-mail: l.eggeling@fz-juelich.de

P. Babu - P. G. Hitchen - H. R. Morris

Division of Molecular Biosciences, Faculty of Natural

Sciences, Imperial College, London SW7 2AZ, UK

H. R. Morris · A. Dell

M-Scan Ltd., Wokingham, Berks RG41 2TZ, UK the lipoglycan possessed an $\alpha$-D-glucopyranosyluronic acid-(1 $\rightarrow 3$ )-glycerol (GlcAGroAc $)_{2}$ based anchor which was then further glycosylated by $8-22$ mannose residues, with $\mathrm{Man}_{12-20}$ GlcAGroAC $_{2}$ molecular species being the most abundant, to form a novel lipomannan structure (Cg-LM-B). The deletion of NCgl2106 in C. glutamicum has now provided a useful strain, in addition with a deletion mutant of NCgl0452 in C. glutamicum for the purification of Cg-LM-A and Cg-LM-B. Interestingly, both Cg-LM species induced a similar production of TNF- $\alpha$ by a human macrophage cell line suggesting that the phospho-myo-inositol residue of the PI-anchor does not play a key role in lipoglycan pro-inflammatory activity.

Keywords Corynebacterium glutamicum . Lipomannan $\cdot$ Mannosyltransferase $\cdot$ PimB $^{\prime}$

\section{Introduction}

The characteristic cell envelope of Corynebacterineae contain mycolic acids, arabinogalactan (AG) and peptidoglycan, which are covalently linked together to form the mycolyl-arabinogalactan-peptidoglycan (mAGP) complex (reviewed in Dover et al. 2004). In addition to the mAGP complex, other glycolipids, such as phosphatidyl-myo-inositol (PI) mannosides (PIMs) and lipoglycans, termed lipomannan (LM) and lipoarabinomannan (LAM) are characteristic of this taxon (Besra et al. 1997). LM and LAM possess 
important physiological and immune functions attributed to a variety of terminal-capping motifs (Nigou et al. 2002), including ManLAM (Chatterjee et al. 1993; Khoo et al. 1995) and PILAM (Gilleron et al. 1997). M. tuberculosis and M. leprae produce ManLAM, which enables them to infect macrophages and dendritic cells (Schlesinger et al. 1994; Tascon et al. 2000). ManLAM inhibits the production of proinflammatory cytokines, such as IL-12 and TNF- $\alpha$ and inhibits phagosomal maturation (Knutson et al. 1998; Nigou et al. 2002; Fratti et al. 2003), while PILAM from a Mycobacterium smegmatis strain induces the proliferation of these cytokines (Adams et al. 1993; Gilleron et al. 1997).

The current model of Corynebacterineae lipoglycan biosynthesis follows two divergent pathways. Firstly, PI $\rightarrow$ PIM $\rightarrow$ LM-A $\rightarrow$ LAM (Besra et al. 1997), and secondly, GroAc ${ }_{2} \rightarrow$ GlcAGroAc $_{2} \rightarrow$ ManGlcAGroAc ${ }_{2} \rightarrow$ LM-B (Tatituri et al. 2007) (Fig. 1). In the first pathway, mycobacterial PI is glycosylated by a $\alpha$-mannopyranosyl (Man $p$ ) residue, a reaction catalyzed by PimA (Rv2610c), which transfers Man $p$ from GDP-mannose to the 2-position of PI to form PIM 1 (Kordulakova et al. 2002). PIM is further acylated (Rv2611c; Kordulakova et al. 2003) and mannosylated by a reaction catalyzed by PimB (Rv0557) resulting in the formation of $\mathrm{Ac}_{1} \mathrm{PIM}_{2}$ (Schaeffer et al. 1999). More recently, however PimB (now also termed MgtA) in C. glutamicum has been shown to be exclusively involved in synthesizing a novel mannosylated glycolipid, 1,2-di- $O-\mathrm{C}_{16} / \mathrm{C}_{18: 1^{-}}$( $\alpha$-D-mannopyranosyl)-(1 $\left.\rightarrow 4\right)$-( $\alpha$-Dglucopyranosyluronic acid)-(1 $\rightarrow 3)$-glycerol (ManGl$\mathrm{cAGroAc}_{2}$ ) (Tatituri et al. 2007). Previous studies have shown that RvD2-ORF1 from M. tuberculosis CDC1551, designated as PimC, catalyzed further $\alpha$-mannosylation of $\mathrm{Ac}_{1} \mathrm{PIM}_{2}$, resulting in $\mathrm{Ac}_{1} \mathrm{PIM}_{3}$ (Kremer et al. 2002). Recently, PimE (Rv1159) has been shown to be involved in higher PIM biosynthesis and directly in the biosynthesis of $\mathrm{Ac}_{1} \mathrm{PIM}_{5}$ (Morita et al. 2006).

The core mannan backbone is glycosylated by Rv2181 and results in the synthesis of $\alpha(1 \rightarrow 2)$ Man $p$ linked branches, characteristic of the mannan backbone in LM and LAM (Kaur et al. 2006). Recently, Mishra et al. (2007) and Kaur et al. (2007) reported a novel $\alpha$-mannosyltransferase, MptA (Rv2174), involved in the latter stages of LM/LAM biosynthesis in Corynebacterineae. The mature LM is then elaborated with arabinose by EmbC to form LAM (Berg et al. 2005). Recently a novel mannosyltransferase, MT1671 (and Rv1635c), has been shown to add terminal Man $p$ residues to the mature LAM in M. tuberculosis to form ManLAM (Dinadayala et al. 2006; Appelmelk et al. 2007).

In this study, we have examined an NCg12106 null mutant of $C$. glutamicum and established that $\mathrm{NCgl} 2106$ is a phosphatidyl-myo-inositol monomannoside mannopyranosyltransferase exclusively involved in the synthesis of $\mathrm{Ac}_{1} \mathrm{PIM}_{2}$ from $\mathrm{Ac}_{1} \mathrm{PIM}_{1}$, now termed Cg-pimB' (Lea-Smith et al. 2008) (Fig. 1). In addition, the utilisation of this mutant strain allowed the isolation and chemical analysis of a novel lipoglycan, now termed Cg-LM-B, based on a GlcAGroAc $c_{2}$ anchor rather than a PI-based anchor, which typifies Corynebacterineae LM and LAM. Moreover, purification of Cg-LM-A and Cg-LM-B, has allowed an evaluation of their pro-inflammatory properties.

\section{Materials and methods}

Strains and culture conditions

The wild type of Corynebacterium glutamicum, ATCC 13032, was grown on either the complex medium Brain Heart Infusion (BHI) (Difco) or a salt medium CGXII (Eggeling and Bott 2005) at $30^{\circ} \mathrm{C}$. The Escherichia coli strain DH5 was grown on LB at $37^{\circ} \mathrm{C}$. Kanamycin and ampicillin were used at a concentration of 25 or $50 \mu \mathrm{g} / \mathrm{ml}$, wherever appropriate. Samples for lipid analysis were prepared by harvesting the cells at an optical density $600 \mathrm{~nm}$ of $10-15$ followed by a saline wash and freeze drying.

Construction of plasmids and strains

To construct the deletion vector $\mathrm{pK} 19$ mobsacB $\Delta 2106$ crossover PCR was applied with primer pairs Nout2106/Nin2106 (Nout2106, AATCGGAGATCC GAGACCGGG; Nin2106, CCCATCCACTAAACTT AAACATTTTCGGGATGCAGACACAAAGA) (all primers in $5^{\prime}-3^{\prime}$ direction) and Cout2106/Cin2106 (Cout2106, ACCCAGTTGTCAGCGCCTTGAG; Cin 2106, TGTTTAAGTTTAGTGGATGGGCGGTTG ACCAATATTTTGCAGAG) with C. glutamicum genomic DNA as template. Both amplified products 
Fig. 1 Schematic representation of MGDAG and PIM pathway for lipoglycan synthesis in Corynebacterianae

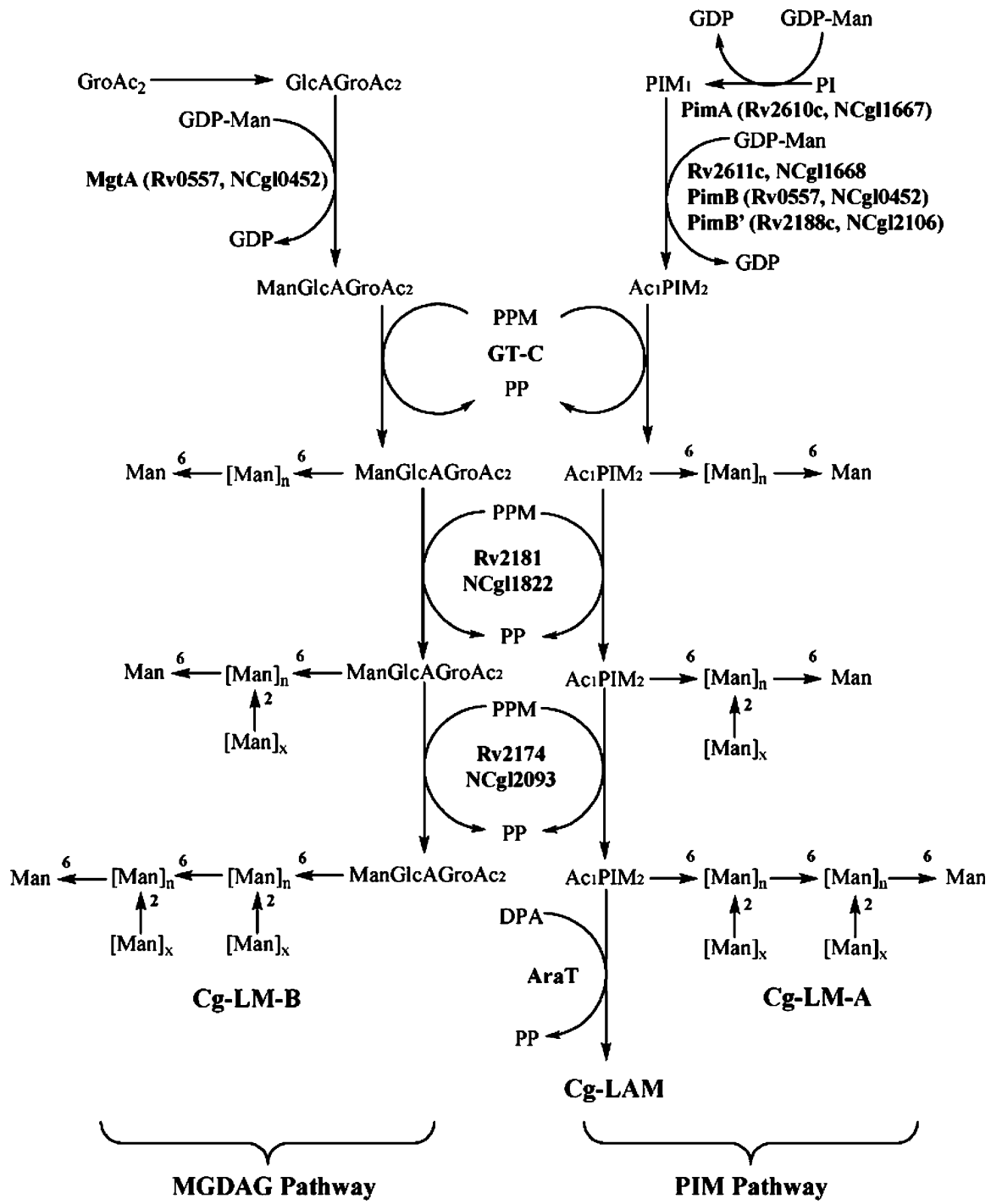

were used in a second PCR with primer pairs Nout2106/Cout2106 to generate a 1025 bp fragment consisting of sequences adjacent to $\mathrm{NCgl} 2106$, which was made blunt, phosphorylated and ligated with SmaI-cleaved pK19mobsacB. The chromosomal deletion of NCgl2106 was performed as described previously using two rounds of positive selection (Schafer et al. 1994), and its successful deletion verified by use of two different primer pairs. Plasmids were introduced into $C$. glutamicum by electroporation with selection to kanamycin resistance $(25 \mu \mathrm{g} / \mathrm{ml})$ on BHI. To enable expression of NCgl2106 in the deletion mutant, NCgl2106 was amplified using the primer pair CGCGGATCCAAGGAG ATATAGATA
TGTCTGCATCCCGAAAAACTCTC and CGCGAA TTCTCATCGTGGTTCACTCTGC. The purified PCR fragment was digested with BamHI-EcoRI and ligated with pEKEx2 (Eikmanns et al. 1991). All cloned fragments were verified by sequencing.

Lipid extraction and purification of lipoglycans

Polar lipids and apolar lipids were extracted as described previously (Dobson et al. 1985). The polar lipid extract was examined by 2D-TLC on aluminum backed plates of silica gel $60 \mathrm{~F}_{254}$ (Merck 5554), using $\mathrm{CHCl}_{3} / \mathrm{CH}_{3} \mathrm{OH} / \mathrm{H}_{2} \mathrm{O}(60: 30: 6$, v/v/v) in the first direction and $\mathrm{CHCl}_{3} / \mathrm{CH}_{3} \mathrm{COOH} / \mathrm{CH}_{3} \mathrm{OH} / \mathrm{H}_{2} \mathrm{O}$ (40:25:3:6, 
$\mathrm{v} / \mathrm{v} / \mathrm{v} / \mathrm{v})$ in the second direction. C. glutamicum glycolipids were visualized by spraying plates with either $\alpha$-naphthol/sulfuric acid, Dittmer \& Lester Reagent, or $5 \%$ ethanolic molybdophosphoric acid followed by gentle charring of plates. Glycolipids were further purified into individual species by preparative TLC on $10 \mathrm{~cm} \times 20 \mathrm{~cm}$ plastic backed TLC plates of silica gel $60 \mathrm{~F}_{254}$ (Merck 5554), run in chloroform/methanol/ water $(60: 30: 6, v / v / v)$. The plates were then sprayed with $0.01 \%$ 1,6-diphenyl-1,3,5-hexatriene dissolved in petroleum ether/acetone $(9: 1 \mathrm{v} / \mathrm{v})$ and the glycolipids visualized under UV light. Following detection the plates were re-developed in toluene to remove diphenyl-1,3,5-hexatriene and the corresponding glycolipid bands were scraped from the plates and extracted from the silica gel using chloroform/ methanol $(2: 1, \mathrm{v} / \mathrm{v})$. Samples were prepared for MALDI-TOF-MS as described previously (Tatituri et al. 2007). Lipoglycans from C. glutamicum strains were extracted and purified as described previously (Nigou et al. 1997; Ludwiczak et al. 2002; Tatituri et al. 2007).

Permethylation of Cg-LM-B prior to MALDI-TOF analysis

Permethylation was performed using the sodium hydroxide procedure as described previously (Dell et al. 1993). MALDI-TOF and TOF/TOF MS data on permethylated samples were acquired in the positive ion mode $(\mathrm{M}+\mathrm{Na})^{+}$using a 4800 (Applied Biosystems) mass spectrometer in the reflector mode with delayed extraction. The collision energy was set to $1 \mathrm{kV}$, and argon was used as collision gas for MS/MS data collection. Samples were dissolved in methanol, and $1 \mu \mathrm{l}$ was mixed at a $1: 1$ ratio $(\mathrm{v} / \mathrm{v})$ with 2,5 dihydrobenzoic acid $(20 \mathrm{mg} / \mathrm{ml}$ in $70 \%$ methanol in water) as matrix.

Gas chromatography-mass spectrometry linkage analysis

Partially methylated alditol acetates were prepared from permethylated samples for gas chromatography-mass spectrometry (GC-MS) linkage analysis as described (Dell et al. 1993). The partially methylated alditol acetates were analyzed using a PerkinElmer Clarus 500 instrument fitted with a RTX-5 column (30 $\mathrm{m} \times 0.25-\mathrm{mm}$ internal diameter, Restek Corp.).
The sample was dissolved in hexane and injected onto the column at $65^{\circ} \mathrm{C}$. The column was maintained at this temperature for $1 \mathrm{~min}$ and then heated to $290^{\circ} \mathrm{C}$ at a rate of $8^{\circ} \mathrm{C}$ per min.

TNF- $\alpha$ production by human macrophage cell line

THP-1 human monocyte/macrophage cell line was maintained in continuous culture with RPMI 1640 medium (Lonza), $10 \%$ fetal calf serum (Lonza) in an atmosphere of $5 \% \mathrm{CO}_{2}$ at $37^{\circ} \mathrm{C}$, as non-adherent cells. Lipoglycans at concentrations of 5 or $15 \mu \mathrm{g} / \mathrm{ml}$ were added in triplicate to cells $\left(10^{5}\right.$ cells/well) in 96 -well culture plates and then incubated for $16 \mathrm{~h}$ at $37^{\circ} \mathrm{C}$. Supernatants were assayed for TNF- $\alpha$ by sandwich ELISA using commercially available kits and according to manufacturer's instructions (R\&D Systems).

\section{Results}

Construction and growth of C. glutamicum $\Delta$ pimB'

We found that the gene product of $\mathrm{NCg10452}$ of C. glutamicum, respectively its ortholog Rv0557 in M. tuberculosis, originally termed PimB and now termed MgtA (Tatituri et al. 2007), primarily acts as an $\alpha$-mannosyltransferase to add mannose to GlcAGro$\mathrm{Ac}_{2}$ (Tatituri et al. 2007). This study also highlighted the presence of a lipomannan, now termed Cg-LM-B, based on a GlcAGroAc ${ }_{2}$ anchor rather than a PI-anchor. Further characterization of Cg-LM-B was hampered due to co-migration and co-elution following size exclusion chromatography with the PI-based Cg-LM-A (Tatituri et al. 2007). To investigate the structure and function of Cg-LM-B further we adopted a strategy based on the existence of two pathways to lipoglycan synthesis (Fig. 1) and to identify Cg-pimB' involved in $\mathrm{Ac}_{1} \mathrm{PIM}_{2}$ synthesis whereby disruption would block $C$. glutamicum LM/LAM synthesis while Cg-LM-B would be unaffected. We searched for unknown glycosyltransferases using nucleotide-activated sugars. Amongst others we identified NCg12106 in the genome of $C$. glutamicum with orthologs present in all Corynebacterianeae including Mycobacterium species.

In order to delete $\mathrm{NCgl} 2106$ in the wild type of C. glutamicum, we constructed the non-replicative 
plasmid pK19mobsacB $\Delta 2106$. This was used to transform C. glutamicum to kanamycin resistance, indicating integration in its chromosome (Fig. 2a). Loss of vector was obtained by selection for sucroseresistance yielding clones with NCg12106 deleted. A PCR analysis with primers hybridizing outside of the sequences used for strain construction resulted in the expected fragment of $2159 \mathrm{bp}$ for the wild type and of $1088 \mathrm{bp}$ for the deletion mutant, which was termed C. glutamicum $\Delta$ pimB'

Polar lipid analysis

Lyophilised cells were extracted using petroleum-ether and methanolic saline to recover apolar lipids. Further processing of the methanolic extract afforded the polar lipid fraction, which was examined by two-dimensional thin-layer chromatography (2D-TLC). The extract from wild type $C$. glutamicum showed the presence of ManGlcAGroAc 2 , GlcAGroAc 2 (Gl-A) and Ac $_{1} \mathrm{PIM}_{2}$, by $\alpha$-naphthol/sulfuric acid staining (Fig. 2b). Surprisingly, while the synthesis of ManGlcAGroAc $c_{2}$ and GlcAGroAc$_{2}$ remained unaffected the component corresponding to $\mathrm{Ac}_{1} \mathrm{PIM}_{2}$ identified by negative ion mode MALDI-MS at $m / z 1398[\mathrm{M}-\mathrm{H}]^{-}$with the fatty acyl groups $\mathrm{C}_{16}$ and $\mathrm{C}_{18: 1}$ (Tatituri et al. 2007) was absent in C. glutamicum $\Delta$ pim $^{\prime}$ with the appearance of a new product, which was sugar (Fig. 2b) and phosphate positive (data not shown) by specific staining. This predominant lipid spot was purified and corresponded to $\mathrm{Ac}_{1} \mathrm{PIM}_{1}$, which was confirmed by negative ion mode MALDI-MS analyses due to the characteristic ion at $\mathrm{m} / \mathrm{z}$ $1236(\mathrm{M}-\mathrm{H})^{-}$(data not shown). Complementation of C. glutamicum $\Delta$ pimB $B^{\prime}$ by pEKEx2-Cg-pimB $B^{\prime}$ restored the wild type phenotype (Fig. 2b). Altogether, the data indicated that $\mathrm{Ac}_{1} \mathrm{PIM}_{1}$ in $C$. glutamicum $\Delta$ pim $B^{\prime}$ occurs possibly as a result of inactivation of a phosphatidylmyo-inositol mannosyltransferase, presumably which transfers a mannopyranosyl (Man $p$ ) residue from GDPMannose to the 6-position of $\mathrm{Ac}_{1} \mathrm{PIM}_{1}$. In addition this data also shed further light on the acylation step in PIM biosynthesis in Corynebacterianeae. The accumulation of $\mathrm{Ac}_{1} \mathrm{PIM}_{1}$ in $C$. glutamicum $\Delta$ pimB $B^{\prime}$ showed that the acylation step (Kordulakova et al. 2003) precedes the second mannosylation step in PIM biosynthesis, and results in the formation of $\mathrm{Ac}_{1} \mathrm{PIM}_{2}$ (Schaeffer et al. 1999).

Lipoglycans were extracted by refluxing delipidated cells in ethanol, followed by hot-phenol extraction, protease digestion and dialysis to remove impurities. The extracted lipoglycans were examined initially on $15 \%$ SDS-PAGE (Fig. 2c). Extracts from wild type $C$. glutamicum showed the presence of Cg-LAM, Cg-LM-A and Cg-LM-B (Tatituri et al. 2007). As expected the lipoglycan extract from C. glutamicum $\Delta$ pim $B^{\prime}$ showed the absence of Cg-LAM and $\mathrm{Cg}-\mathrm{LM}-\mathrm{A}$, and the presence of a single species (Cg-LM-B) by chemical characterization as described below. Complementation of $C$. glutamicum $\Delta$ pimB $^{\prime}$ by transformation with plasmid pEKEx2-Cg-pimB $B^{\prime}$ restored the wild type phenotype of Cg-LAM, Cg-LM-A and Cg-LM-B (Fig. 2c). As predicated, the inactivation of $\mathrm{Cg}-\mathrm{PimB}^{\prime}$ abolished the synthesis of Cg-LAM and Cg-LM-A, and provided a strain that allowed the purification of Cg-LM-B for further chemical and functional characterisation. Cg-LM-B from C. glutamicum $\Delta$ pimB $B^{\prime}$ and Cg-LM-A from C. glutamicum $\Delta$ pimB' (Tatituri et al. 2007) were purified by hydrophobic interaction chromatography (HIC) and size exclusion chromatography.

Analysis of Cg-LM-B by mass spectrometry

The Cg-LM-B was permethylated prior to detailed mass spectrometric analysis. During the permethylation step the acyl groups from the lipoglycan are liberated and the glycerol hydroxyl groups are methylated. The MALDI-TOF MS profile of the permethylated sample in the positive mode showed no signals for ManGlcAGro $(\mathrm{m} / z, 579)$ but, interestingly, a series of peaks $\left(\mathrm{M}+\mathrm{Na}^{+}\right)$corresponding to the addition of 4-22 hexose residues to the GlcAGro core structure. In the lower mass region of the spectrum, signals consistent with the elongation of the GlcAGro core were observed at $\mathrm{m} / \mathrm{z}$ 1191.7, 1395.8 and 1600.0 corresponding to the compositions $\mathrm{Hex}_{4}$ GlcAGro, Hex 5 GlcAGro and $\mathrm{Hex}_{6}$ GlcAGro, respectively (data not shown). Peaks corresponding to $\mathrm{Hex}_{7}$ GlcAGro and Hex ${ }_{9}$ GlcAGro were absent from the spectrum. At higher mass, signals were observed corresponding to a series of $\mathrm{Hex}_{n}$ GlcAGro units containing even numbers of hexoses at $\mathrm{m} / \mathrm{z}$ 2008.2, 2416.4, 2824.6, 3232.8, 3641.0, 4049.2, 4457.2 and 4865.6 with Hex $_{12}$ GlcAGro $(\mathrm{m} / \mathrm{z}$ 2824.6) being most abundant (Fig. 3a). The low abundance of signals attributable to components carrying an odd number (Hex11, 13, 15, 17, 19 and $21)$ of hexoses $(\mathrm{m} / \mathrm{z}, 2620.5,3028.7,3436.9,3849.0$, 
Fig. 2 Construction of inframe deletion mutant of pimB $B^{\prime}$ in $C$. glutamicum and resulting phenotype. (a) Strategy to delete $\operatorname{pim}^{\prime}$ by use of vector pK19mobsacB $\Delta$ pimB' by two homologous recombination events with the wild type chromosome C. glutamicum (Cg-WT).

The deletion is demonstrated on the right via PCR using primer pairs P5/P6 showing the expected $1088 \mathrm{bp}$ fragment for the deletion mutant in the lane marked " $\Delta$ ", and that of $2159 \mathrm{bp}$ for the wild type marked "W". The lane marked "St" is the standard consisting of BstEIIfragments of $\lambda$-DNA, with arrowheads positioned at $0.70,1.37,2.32$, and $3.68 \mathrm{~kb}$. (b) TLC-analysis of PIM biosynthesis in C. glutamicum,

C. glutamicum $\Delta$ pimB $B^{\prime}$ and C. glutamicum $\Delta$ pim $B^{\prime}$ pEKEx2-pim $B^{\prime}$. Glycolipids were visualized by spraying plates with $\alpha$-naphthol/ sulfuric acid, followed by gentle charring of TLC plates. (c) Lipoglycan profiles of C. glutamicum strains analyzed using SDSPAGE and visualized using a Pro-Q emerald glycoprotein stain (Invitrogen) specific for carbohydrates. The major bands represented by $\mathrm{Cg}$ LAM, Cg-LM-A, and CgLM-B are indicated. The four major standard bands indicated on the side of the gel represent glycoproteins of $180,82,42$ and $18 \mathrm{kDa}$, respectively
A
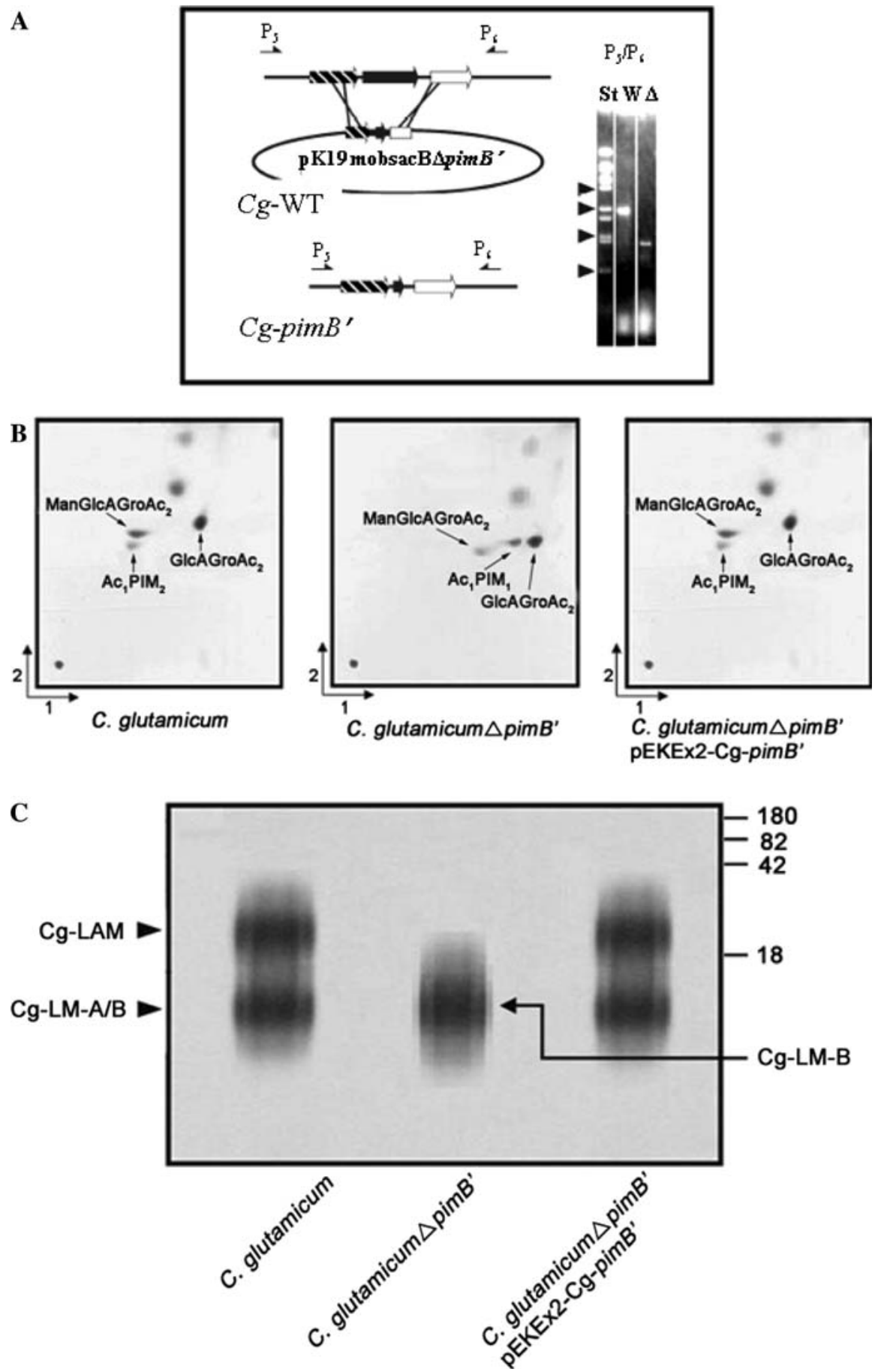

4252.2 and 4660.4), as compared to even numbered, suggests that the hexose polymer is branched rather than linear. In addition, there are also signals present in the MS spectrum attributable to hexose oligomers. This may result from partial degradation of the sample during preparation but could also be due to contamination. Each of the major signals in the spectrum was subjected to collision induced decomposition MS/MS (CID-MS/MS) analysis to establish their structures. For example, MS/MS spectra of the 
signal at $\mathrm{m} / \mathrm{z}$ 2416, which has the composition Hex $_{10}$ GlcAGro, showed peaks at $\mathrm{m} / \mathrm{z}$ 2198, 1994, 1585.6, 1381.5, 1177.5, 769.3 and 361 (Fig. 3b) which are due to the loss of $1,2,4,5,6,8$ and 10 hexose units from the molecular ion. The inset to Fig. $3 b$ shows the likely sequence that is consistent with this set of fragment ions.

In order to confirm the nature of the hexose units and linkages, GC/MS analysis of partially methylated alditol acetates was carried out. This revealed the presence of terminal mannose, 2 linked mannose, 2,6 linked mannose (data not shown). The presence of
t-Man and 2,6-Man convincingly establishes that the backbone of the oligomannans is heavily branched. The relative abundances of t-Man (1.0), 6-Man (0.5) and 2,6-Man (0.98) are consistent with single mannose residues being appended at O-2 to the 6-linked mannosyl backbone.

TNF- $\alpha$ production by human macrophage cell line in response to $\mathrm{Cg}$-LMs

PI-anchored lipoglycans, and most particularly LMs, isolated from other actinomycete genera, including
Fig. 3 MALDI-TOF/TOF analysis of permethylated Cg-LM-B $\left[\mathrm{M}+\mathrm{Na}^{+}\right]$ Man $_{n}$ GlcAGro. (a) MS spectrum of the dervatized Cg-LM-B. Unassigned peaks (*) are due to permethylation artifacts. (b) CID-MS/MS analysis of $m / z, 2416\left(\mathrm{M}+\mathrm{Na}^{+}\right)$ Man $_{10}$ GlcAGroAc. A possible structure and CID fragmentation pattern of peak $m / z, 2416$ is depicted in the cartoon representation, inset. Circle-mannose; diamond-glucuronic acid
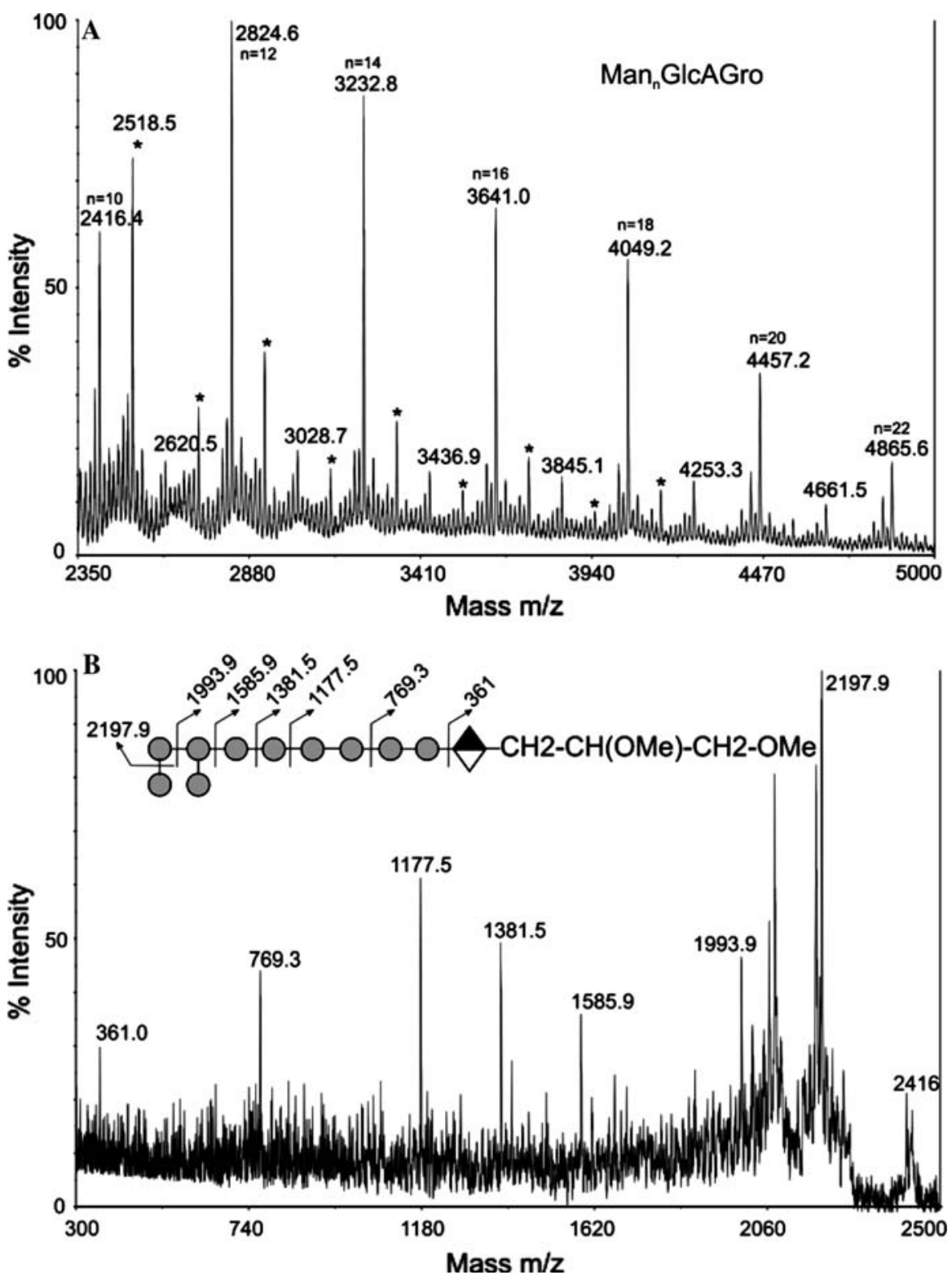
mycobacteria, have previously been shown to induce cytokine production by phagocytic cells (Garton et al. 2002; Gibson et al. 2004; Quesniaux et al. 2004). We thus investigated the potency of both types of $\mathrm{Cg}$-LMs to stimulate the release of TNF- $\alpha$ using a human macrophage cell line. As expected, the PI-anchored LM, Cg-LM-A, elicited a dose-dependent production of the cytokine (Fig. 4). However interestingly, the GlcAGroAc $\mathrm{A}_{2}$-anchored LM, $\mathrm{Cg}-\mathrm{LM}-\mathrm{B}$, was also found to be as stimulatory as the PI-anchored LM, suggesting that the phosphomyo-inositol unit of the anchor in LM does not play a key role in lipoglycan pro-inflammatory activity.

\section{Discussion}

SDS-PAGE analysis of purified lipoglycans from C. glutamicum $\Delta$ pimB $^{\prime}$ showed the presence of a single species, which migrated akin to $\mathrm{Cg}$-LM. Chemical characterization of this novel species using MS-MS established it as an oligomer of hypermannosylated oligosaccharides linked to GlcAGroAc 2 . It was previously established that these glycosylated diacylglycerols function as precursors/anchors for

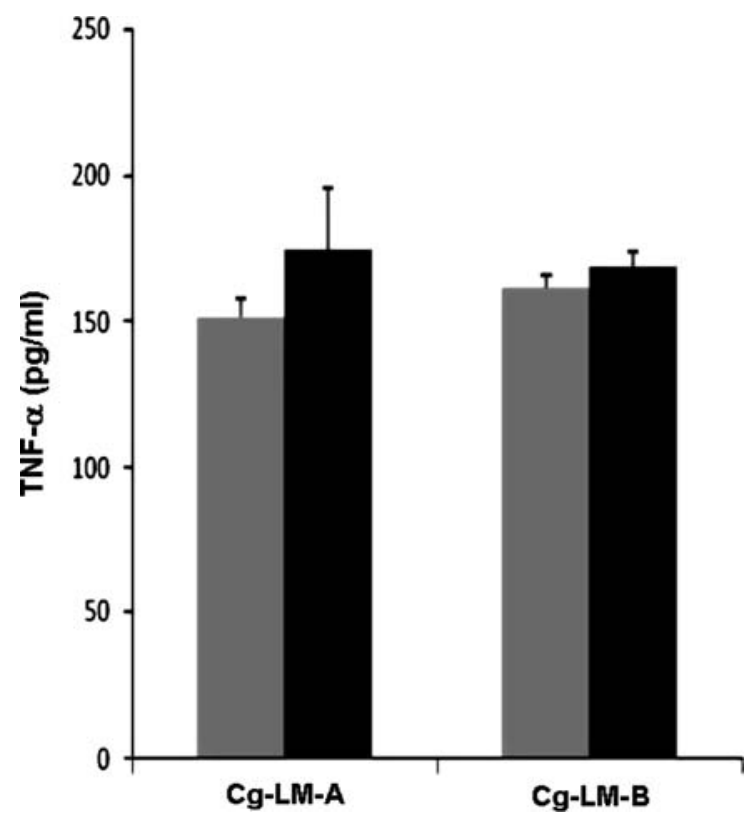

Fig. 4 TNF- $\alpha$ production by human macrophage cell line in response to $\mathrm{Cg}-\mathrm{LMs}$. $\mathrm{Cg}$-LM-A was isolated and purified as described previously (Tatituri et al. 2007) and Cg-LM-B (this study) were tested at 5 (grey bars) and 15 (black bars) $\mu \mathrm{g} / \mathrm{ml}$ hyperglycosylated variants, such as the lipomannans, as found in the case of dimannosyl diacylglycerols in Micrococcus and lipoteichoic acids (Pakkiri and Waechter 2005). Moreover, related di- and monoacylglycerols containing glucuronosyl residues have been well reported in Pseudomonas sp. (Wilkinson 1968) Bacillus cereus T (Minnikin et al. 1971), and halotolerant bacteria (Stern and Tietz 1971). The data in relation to Mycobacterium are sparse in regards to the presence of glycosyl diacylglycerols, except for a few limited cases, such as a diglucosyl diacylglycerol (Hunter et al. 1986). In Mycobacterium smegmatis, a minor glucuronosyl diacylglycerol glycolipid has been reported (Wolucka et al. 1993), and uronosylcontaining glycopeptidolipids in Mycobacterium avium (Chatterjee et al. 1987) and Mycobacterium habana (Khoo et al.1996). Our results also support our initial hypothesis that ManGlcAGroAc 2 also participates in the biosynthesis of a novel Cg-LMlike molecule and that the Cg-LM most likely consists of two components, a minor $\mathrm{Cg}-\mathrm{LM}$ based on ManGlcAGroAc $_{2}$ (Cg-LM-B) and a more abundant component akin to the characteristic dominant mycobacterial PI-based LM (Cg-LM-A) (Tatituri et al. 2007). The presence of a Cg-LM-B type species may exist in Mycobacterium, based on the identification as mentioned earlier of a minor glucuronosyl diacylglycerol glycolipid (Wolucka et al. 1993), and further experiments are needed to address this question. It is also interesting to also note that $\mathrm{Cg}$-LM-A is arabinosylated, yet Cg-LM-B, which possesses a similar mannan is not arabinosylated. A possibility for this structural difference maybe that $\mathrm{Cg}-\mathrm{LM}-\mathrm{B}$ is more heavily branched than Cg-LM-A thereby removing potential sites for arabinosylation leading to Cg-LAM.

LM-like molecules are powerful pro-inflammatory lipoglycans found in the cell-walls of mycobacteria and some related actinomycetes genera (Garton et al. 2002; Gibson et al. 2004; Quesniaux et al. 2004), including Corynebacterium (Tatituri et al. 2007). They are composed of two parts, a PI anchor to which is attached an $\alpha$-D-mannan domain, that both play a key role in their ability to induce cytokine production by phagocytic cells. The PI-acylation pattern is also critical for activity (Gilleron et al. 2006) and the latter is abrogated after deacylation of LM by alkaline treatment (Gibson et al. 2005). But the structure of the glycosidic moiety has also been shown to modulate the pro-inflammatory activity of 
the molecule. Indeed, the presence of an arabinan domain such as in the case of LAM-like molecules undermines lipoglycan activity by masking the mannan domain (Vignal et al. 2003; Gibson et al. 2004). In addition, LM activity seems also to be modulated by the presence or not of side chains on the mannan domain as well as the size of these chains (Gibson et al. 2005). We have shown that C. glutamicum contains two types of LM that share a similar mannan domain but differ by their lipidic anchor, one bearing a classical PI anchor and the other one based on a GlcAGroAc $_{2}$ anchor (Tatituri et al. 2007). In the present study, aided by two different mutants, which are selectively affected in the biosynthesis pathway of each type of LM, we have managed to separate them. We found that both LMs induced a similar amount of cytokine secretion by a human macrophage cell line, indicating that the PI part of Cg-LM-A does not play a key role in lipoglycan proinflammatory activity. Since the completion of these studies and preparation of manuscript, Lea-Smith et al. (2008) have reported similar findings on the role of $\mathrm{Cg}-\mathrm{PimB}^{\prime}$. However, their study did not analyze the structural basis of Cg-LM-B in detail and report on the pro-inflammatory properties of this lipoglycan in comparison to $\mathrm{Cg}-\mathrm{LM}-\mathrm{A}$, which is now reported in this present study. Finally, a proposed hypothetical pathway that takes into account the potential role of MgtA and $\mathrm{PimB}^{\prime}$, the accumulation of $\mathrm{Ac}_{1} \mathrm{PIM}_{1}$ and $\mathrm{Cg}-\mathrm{LM}-\mathrm{B}$, and abrogation of ManGlcAGroAc 2 (Tatituri et al. 2007) and $\mathrm{Ac}_{1} \mathrm{PIM}_{2}$ is presented in Fig. 1.

Acknowledgments AKM is a Darwin Trust sponsored Ph.D. student. GSB acknowledges support in the form of a Personal Research Chair from Mr. James Bardrick, Royal Society Wolfson Research Merit Award, as a former Lister InstituteJenner Research Fellow, the Medical Research Council and The Wellcome Trust (081569/Z/06/Z). A.D. and H.R.M. are supported by funding from the Biotechnology and Biological Sciences Research Council and the Wellcome Trust. A.D. was a Biotechnology and Biological Sciences Research Council Professorial Research Fellow. P.B. thanks the UK-India Education Research Initiative for a Research Fellowship. We are thankful for technical assistance provided by Peter Ashton for MALDI-MS analysis and Jerome Nigou for cytokine assays.

Open Access This article is distributed under the terms of the Creative Commons Attribution Noncommercial License which permits any noncommercial use, distribution, and reproduction in any medium, provided the original author(s) and source are credited.

\section{References}

Adams LB, Fukutomi Y, Krahenbuhl JL (1993) Regulation of murine macrophage effector functions by lipoarabinomannan from mycobacterial strains with different degrees of virulence. Infect Immun 61:4173-4181

Appelmelk BJ, den Dunnen J, Driessen NN et al (2007) The mannose cap of mycobacterial lipoarabinomannan does not dominate the Mycobacterium-host interaction. Cell Microbiol (in press)

Berg S, Starbuck J, Torrelles JB, Vissa VD, Crick DC, Chatterjee D, Brennan PJ (2005) Roles of conserved proline and glycosyltransferase motifs of EmbC in biosynthesis of lipoarabinomannan. J Biol Chem 280:5651-5663

Besra GS, Morehouse CB, Rittner CM, Waechter CJ, Brennan PJ (1997) Biosynthesis of mycobacterial lipoarabinomannan. J Biol Chem 272:18460-18466

Chatterjee D, Aspinall GO, Brennan PJ (1987) The presence of novel glucuronic acid-containing, type-specific glycolipid antigens within Mycobacterium sp. Revision of earlier structures. J Biol Chem 262:3528-3533

Chatterjee D, Khoo KH, McNeil MR, Dell A, Morris HR, Brennan PJ (1993) Structural definition of the nonreducing termini of mannose-capped LAM from Mycobacterium tuberculosis through selective enzymatic degradation and fast atom bombardment-mass spectrometry. Glycobiology 3:497-506

Dell A, Khoo KH, Panico M, McDowell RA, Etienne AT, Reason AJ, Morris HR (1993) FAB-MS and ES-MS of glycoproteins. In: Fukuda M, Kobata A (eds) Glycobiology: a practical approach. Oxford University Press, Oxford (UK), pp 187-222

Dinadayala P, Kaur D, Berg S, Amin AG, Vissa VD, Chatterjee D, Brennan PJ, Crick DC (2006) Genetic basis for the synthesis of the immunomodulatory mannose caps of lipoarabinomannan in Mycobacterium tuberculosis. J Biol Chem 281:20027-20035

Dobson G, Minnikin DE, Minnikin SM, Parlett JH, Goodfellow M, Ridell M, Magnusson M (1985) Systematic analysis of complex mycobacterial lipids. In: Goodfellow M, Minnikin DE (eds) Chemical methods in bacterial systematics. Academic Press, London (UK), pp 237-265

Dovser LG, Cerdeno-Tarraga AM, Pallen MJ, Parkhill J, Besra GS (2004) Comparative cell wall core biosynthesis in the mycolated pathogens, Mycobacterium tuberculosis and Corynebacterium diphtheriae. FEMS Microbiol Rev 28:225-250

Eggeling L, Bott M (2005) Handbook of Corynebacterium glutamicum. CRC Press, Taylor Francis Group

Eikmanns BJ, Kleinertz E, Liebl W, Sahm H (1991) A family of Corynebacterium glutamicum/Escherichia coli shuttle vectors for cloning, controlled gene expression, and promoter probing. Gene 102:93-98

Fratti RA, Chua J, Vergne I, Deretic V (2003) Mycobacterium tuberculosis glycosylated phosphatidylinositol causes phagosome maturation arrest. Proc Natl Acad Sci U S A 100:5437-5442

Garton NJ, Gilleron M, Brando T, Dan HH, Giguère S, Puzo G, Prescott JF, Sutcliffe IC (2002) A novel lipoarabinomannan from the equine pathogen Rhodococcus equi. 
Structure and effect on macrophage cytokine production. J Biol Chem 277:31722-31733

Gibson KJ, Gilleron M, Constant P, Brando T, Puzo G, Besra GS, Nigou J (2004) Tsukamurella paurometabola lipoglycan, a new lipoarabinomannan variant with proinflammatory activity. J Biol Chem 279:22973-22982

Gibson KJ, Gilleron M, Constant P, Sichi B, Puzo G, Besra GS, Nigou J (2005) A lipomannan variant with strong TLR-2dependent pro-inflammatory activity in Saccharothrix aerocolonigenes. J Biol Chem 280:28347-28356

Gilleron M, Himoudi N, Adam O, Constant P, Venisse A, Riviere M, Puzo G (1997) Mycobacterium smegmatis phosphoinositols-glyceroarabinomannans. Structure and localization of alkali-labile and alkali-stable phosphoinositides. J Biol Chem 272:117-124

Gilleron M, Nigou J, Nicolle D, Quesniaux V, Puzo G (2006) The acylation state of mycobacterial lipomannans modulates innate immunity response through toll-like receptor 2. Chem Biol 13:39-47

Hunter SW, Gaylord H, Brennan PJ (1986) Structure and antigenicity of the phosphorylated lipopolysaccharide antigens from the leprosy and tubercle bacilli. J Biol Chem 261:12345-12351

Kaur D, Berg S, Dinadayala P, Gicquel B, Chatterjee D, McNeil MR, Vissa VD, Crick DC, Jackson M, Brennan PJ (2006) Biosynthesis of mycobacterial lipoarabinomannan: role of a branching mannosyltransferase. Proc Natl Acad Sci U S A 103:13664-13669

Kaur D, McNeil MR, Khoo KH, Chatterjee D, Crick DC, Jackson M, Brennan PJ (2007) New insights into the biosynthesis of mycobacterial lipomannan arising from deletion of a conserved gene. J Biol Chem 282:2713327140

Khoo KH, Dell A, Morris HR, Brennan PJ, Chatterjee D (1995) Inositol phosphate capping of the nonreducing termini of lipoarabinomannan from rapidly growing strains of Mycobacterium. J Biol Chem 270:12380-12389

Khoo KH, Chatterjee D, Dell A, Morris HR, Brennan PJ, Draper P (1996) Novel O-methylated terminal glucuronic acid characterizes the polar glycopeptidolipids of $\mathrm{Myco}$ bacterium habana strain TMC 5135. J Biol Chem 271:12333-12342

Knutson KL, Hmama Z, Herrera-Velit P, Rochford R, Reiner NE (1998) Lipoarabinomannan of Mycobacterium tuberculosis promotes protein tyrosine dephosphorylation and inhibition of mitogen-activated protein kinase in human mononuclear phagocytes. Role of the Src homology 2 containing tyrosine phosphatase 1. J Biol Chem 273:645-652

Kordulakova J, Gilleron M, Mikusova K, Puzo G, Brennan PJ, Gicquel B, Jackson M (2002) Definition of the first mannosylation step in phosphatidylinositol mannoside synthesis. PimA is essential for growth of mycobacteria. J Biol Chem 277:31335-31344

Kordulakova J, Gilleron M, Puzo G, Brennan PJ, Gicquel B, Mikusova K, Jackson M (2003) Identification of the required acyltransferase step in the biosynthesis of the phosphatidylinositol mannosides of Mycobacterium species. J Biol Chem 278:36285-36295

Kremer L, Gurcha SS, Bifani P, Hitchen PG, Baulard A, Morris HR, Dell A, Brennan PJ, Besra GS (2002) Characterization of a putative $\alpha$-mannosyltransferase involved in phosphatidylinositol trimannoside biosynthesis in Mycobacterium tuberculosis. Biochem J 363:437-447

Lea-Smith DJ, Martin KL, Pyke JS, Tull D, McConville MJ, Coppel RL, Crellin, PK (2008) Analysis of a new mannosyltransferase required for the synthesis of phosphotidylinositol mannosides and lipoarabinomannan revels two lipomannan pools in Corynebacterineae. J Biol Chem (in press)

Ludwiczak P, Gilleron M, Bordat Y, Martin C, Gicquel B, Puzo G (2002) Mycobacterium tuberculosis phoP mutant: lipoarabinomannan molecular structure. Microbiology 148:3029-3037

Minnikin DE, Abdolrahimzadeh H, Baddiley J (1971) The interrelation of polar lipids in bacterial membranes. Biochim Biophys Acta 249:651-655

Mishra AK, Alderwick LJ, Rittmann D, Tatituri RV, Nigou J, Gilleron M, Eggeling L, Besra GS (2007) Identification of an $\alpha(1-6)$ mannopyranosyltransferase (MptA), involved in Corynebacterium glutamicum lipomanann biosynthesis, and identification of its orthologue in Mycobacterium tuberculosis. Mol Microbiol 65:1503-1517

Morita YS, Sena CB, Waller RF, Kurokawa K, Sernee MF, Nakatani F, Haites RE, Billman-Jacobe H, McConville MJ, Maeda Y, Kinoshita T (2006) PimE is a polyprenolphosphate-mannose-dependent mannosyltransferase that transfers the fifth mannose of phosphatidylinositol mannoside in mycobacteria. J Biol Chem 281:25143-25155

Nigou J, Gilleron M, Cahuzac B, Bounery JD, Herold M, Thurnher M, Puzo G (1997) The phosphatidyl-myo-inositol anchor of the lipoarabinomannans from Mycobacterium bovis bacillus Calmette Guerin. Heterogeneity, structure, and role in the regulation of cytokine secretion. J Biol Chem 272:23094-23103

Nigou J, Gilleron M, Rojas M, Garcia LF, Thurnher M, Puzo G (2002) Mycobacterial lipoarabinomannans: modulators of dendritic cell function and the apoptotic response. Microbes Infect 4:945-953

Pakkiri LS, Waechter CJ (2005) Dimannosyldiacylglycerol serves as a lipid anchor precursor in the assembly of the membrane-associated lipomannan in Micrococcus luteus. Glycobiology 15:291-302

Quesniaux VJ, Nicolle DM, Torres D, Kremer L, Guérardel Y, Nigou J, Puzo G, Erard F, Ryffel B (2004) Tolllike receptor 2 (TLR2)-dependent-positive and TLR2 independent-negative regulation of proinflammatory cytokines by mycobacterial lipomannans. J Immunol 172: 4425-4434

Schaeffer ML, Khoo KH, Besra GS, Chatterjee D, Brennan PJ, Belisle JT, Inamine JM (1999) The pimB gene of Mycobacterium tuberculosis encodes a mannosyltransferase involved in lipoarabinomannan biosynthesis. J Biol Chem 274:31625-31631

Schafer A, Tauch A, Jager W, Kalinowski J, Thierbach G, Puhler A (1994) Small mobilizable multi-purpose cloning vectors derived from the Escherichia coli plasmids pK18 and pK19: selection of defined deletions in the chromosome of Corynebacterium glutamicum. Gene 145:69-73

Schlesinger LS, Hull SR, Kaufman TM (1994) Binding of the terminal mannosyl units of lipoarabinomannan from a virulent strain of Mycobacterium tuberculosis to human macrophages. J Immunol 152:4070-4079 
Stern N, Tietz A (1971) Biosynthesis of glucuronosyl diglyceride by a cell-free system obtained from a moderately halophilic-halotolerant bacterium. FEBS Lett 19:217-220

Tascon RE, Soares CS, Ragno S, Stavropoulos E, Hirst EM, Colston MJ (2000) Mycobacterium tuberculosis-activated dendritic cells induce protective immunity in mice. Immunology 99:473-480

Tatituri RV, Illarionov PA, Dover LG, Nigou J, Gilleron M, Hitchen P, Krumbach K, Morris HR, Spencer N, Dell A, Eggeling L, Besra GS (2007) Inactivation of Corynebacterium glutamicum $\mathrm{NCg10452}$ and the role of MgtA in the biosynthesis of a novel mannosylated glycolipid involved in lipomannan biosynthesis. J Biol Chem 282:4561-4572
Vignal C, Guérardel Y, Kremer L, Masson M, Legrand D, Mazurier J, Elass E (2003) Lipomannans, but not lipoarabinomannans, purified from Mycobacterium chelonae and Mycobacterium kansasii induce TNF- $\alpha$ and IL-8 secretion by a CD14-toll-like receptor 2-dependent mechanism. J Immunol 171:2014-2023

Wilkinson SG (1968) Glycolipids containing glucose and uronic acids in Pseudomonas species. Biochim Biophys Acta 152:227-229

Wolucka BA, McNeil MR, Kalbe L, Cocito C, Brennan PJ (1993) Isolation and characterization of a novel glucuronosyl diacylglycerol from Mycobacterium smegmatis. Biochim Biophys Acta 1170:131-136 